\title{
Consistency over time of animal-based welfare indicators as a further step for developing a welfare assessment monitoring scheme: The case of the Animal Welfare Indicators protocol for dairy goats
}

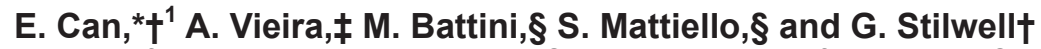 \\ *Division of Agricultural and Environmental Sciences, University of Nottingham, Sutton Bonington Campus, College Road, Sutton Bonington, \\ Loughborough LE12 5RD, United Kingdom \\ †Faculdade de Medicina Veterinária, Animal Behaviour and Welfare Research Lab, Centre for Interdisciplinary Research \\ in Animal Health (CIISA), Universidade de Lisboa, Polo Alto da Ajuda, 12 1300-477, Lisbon, Portugal \\ ‡Centre for Management Studies, Instituto Superior Técnico, Universidade de Lisboa, Avenida Rovisco Pais, 1049-001, Lisbon, Portugal \\ $\S$ Laboratorio di Benessere Animale, Etologia Applicata e Produzioni Sostenibili, Dipartimento di Medicina Veterinaria, \\ Università degli Studi di Milano, Via G. Celoria 10, 20133 Milan, Italy
}

\begin{abstract}
Consistency over time (COT) of animal-based indicators is key to a reliable and feasible welfare protocol, indicating that results are representative over long-term situations. High levels of consistency ensure fairness for the farmer and credibility of the system. In addition, indicator COT reduces recording costs, as having indicators that do not change over a long period of time will require less farm visits to achieve reliable estimates. To date, COT of animal-based indicators included in the welfare assessment of dairy goats has never been tested. Therefore, our aim was to investigate COT of animal-based indicators included in the Animal Welfare Indicators (AWIN) welfare assessment prototype protocol for dairy goats. To meet this goal, a study was designed where an average of 3 mo elapsed between 2 sets of visits to the same 20 dairy goat farms (10 in Portugal and 10 in Italy), with no major changes in management routines or housing conditions occurring during this period. Initially, we performed a Wilcoxon signed rank test to investigate whether the results obtained during the 2 visits were significantly different. After this preliminary screening, the indicators presenting nonsignificant differences between visits were submitted to a second step analysis, where discriminative and evaluative analyses were conducted to reach a final indicator lineup. The discriminative approach helped distinguishing among farms, whereas the agreement analysis showed us the range of differences between repeated assessments. Some particular conclusions could be drawn from this combined analysis, helping to the development of the final AWIN
\end{abstract}

Received March 2, 2017.

Accepted July 21, 2017.

${ }^{1}$ Corresponding author: edna.can@nottingham.ac.uk welfare assessment protocol for dairy goats and as a further step to develop a welfare assessment monitoring scheme for this and other species. In this sense, the AWIN welfare assessment protocol allows for the quick differentiation between farms based on the identification of persistent welfare problems, by recording highly consistent and feasible indicators. In a second step, a more comprehensive protocol, consisting of indicators more likely to be subject to variations along time, was applied. Repeated assessments and long-term studies of indicator consistency are needed to help determine the frequency of visits required to obtain a consistent and feasible welfare assessment scheme. This paper adds to the literature by providing guidance on the variability of animal-based indicators over time.

Key words: animal-based indicator, consistency over time, dairy goat, test-retest reliability

\section{INTRODUCTION}

The implementation of an effective welfare assessment system is essential for the process of improving animal welfare. To be effectively used on different farms, a welfare assessment tool must comprise indicators that are not only valid and feasible, but also reliable (Waiblinger et al., 2001). Reliability is a central aspect when selecting indicators for welfare assessment and can be explored by means of inter- and intraobserver reliability and test-retest reliability. In the current paper, the focus is set on test-retest reliability, which refers to the agreement between observations performed on the same individual on at least 2 different occasions (Scott et al., 2001). A particular case of test-retest reliability is the consistency over time (COT) of animal-based indicators, meaning that results must represent a longterm farm condition instead of being too sensitive to changes, provided that the circumstances have not changed significantly (Knierim and Winckler, 2009; 
Plesch et al., 2010). Consistency over time addresses the major concern stemming from the potential variability of animal welfare indicators across different time periods and environmental conditions.

Consistency over time can be used as an umbrella term for the agreement and reliability concepts, which are often applied interchangeably and expressed by different measures in the literature (de Vet et al., 2006; Terwee et al., 2007). Reliability refers to the extent to which units (e.g., farms) can be distinguished from each other, regardless of measurement error (Streiner and Norman, 2003). High reliability is important when considering a discriminative approach (i.e., if we want to distinguish among units). On the other hand, agreement relates to the absolute measurement error and to how close the results on repeated measurements are. A small measurement error is thus necessary for an evaluative perspective, allowing for the distinction of important changes from measurement error (Terwee et al., 2007). In general, reliability measures can be considered necessary for methods that are used for discriminative purposes, whereas agreement measures are mostly required for those that are applied from an evaluative perspective (Guyatt et al., 1987; de Vet et al., 2006).

No gold standard exists to measure COT of animal welfare indicators, and several measures of reliability and agreement can be found in the literature [e.g., Spearman's rank correlation $\left(\mathbf{r}_{\mathrm{s}}\right)$, intraclass correlation coefficient (ICC), limits of agreement (LoA)]. However, the interpretation of reliability of continuous variables should be performed carefully, as inconsistencies in the analysis of results obtained with different methods have been reported (Costa-Santos et al., 2011). A potential approach to overcome these inconsistencies relies on the combined use of reliability and agreement measures (de Vet et al., 2006; Dohoo et al., 2009).

Different studies have investigated COT of several animal-based welfare indicators (e.g., O'Callaghan et al., 2003; Winckler et al., 2007; Bokkers et al., 2009), and of those included in the Welfare Quality protocols for beef cattle (e.g., Kirchner et al., 2014) and for growing pigs (e.g., Temple et al., 2013; Czycholl et al., 2016); Temple et al. (2013) combined the use of reliability and agreement measures. Nevertheless, to date, COT of indicators used for the welfare assessment of dairy goats has never been tested. Therefore, the aim of our study was to investigate COT of animal-based indicators included in the Animal Welfare Indicators (AWIN) welfare assessment prototype protocol for intensively kept dairy goats as a further step to develop a reliable welfare assessment monitoring scheme for this species.

\section{MATERIALS AND METHODS}

\section{Farm Sample and Data Collection}

Data collection was carried out on 20 intensive dairy goat farms located in Portugal $(\mathrm{n}=10)$ and Italy ( $\mathrm{n}$ $=10$ ). In Portugal, farms were randomly selected from the national database of Direcção-Geral de Alimentação e Veterinária (Portuguese veterinary services, Lisbon); in Italy, farms were selected with the support of the S.A.T.A. (Technical Advice Service for Farmers) of the Lombardy region (Milan). The number of animals per farm ranged from 18 (Italy) to 2,000 dairy goats (Portugal), with a mean $( \pm \mathrm{SD})$ of $362( \pm 480)$ adult dairy goats, which were kept indoors on concrete, soil, or grit covered with straw as bedding material. In Portugal, the number of dairy goats on each farm ranged from 50 to 2,000 animals, with a mean of $522( \pm 584)$ goats; in Italy, the number of dairy goats ranged from 18 to 912 animals, with a mean of $202( \pm 280)$ goats. To investigate the animal welfare indicators' COT, each farm was visited between February and March 2014 (winter period) and then revisited between June and July 2014 (summer period) by the same assessor who performed the first assessment. An average of 4 mo $(3.7 \pm 1.0 \mathrm{mo})$ elapsed between visits, and no major alterations in management and housing conditions were implemented during this period. Major alterations were defined as alterations on the management or resources that exceed changes that could be expected over time (Kirchner et al., 2014). However, routine changes as isolation of sick animals, culling, and replacement of goats were maintained. The animals' lactation stage obviously changed as well.

All assessments followed the welfare assessment prototype protocol developed by the AWIN project for lactating dairy goats in intensive husbandry systems, which comprises 25 animal-based indicators structured in accordance with the 4 principles and 12 criteria developed by Welfare Quality [Botreau et al. (2007a); for details on the description of animal-based indicators see Battini et al. (2015, 2016) and Can et al. (2016)]. The AWIN prototype protocol combines 14 indicators at group level and 11 indicators at individual level, which are assessed to produce each indicator's prevalence. Assessments began with group-level observations of the number of goats improperly disbudded, queuing at feeding or drinking place, poor hair coat condition, showing oblivion behavior or signs of thermal stress (either shivering or panting), and abnormally lying in the pen or kneeling at the feeding rack. Immediately after this assessment, a qualitative behavior assessment was conducted. Subsequently, the assessor entered the 
pen, and human-animal relationship tests [latency to the first contact and avoidance distance (AD) tests] were carried out. Finally, observations of individual animals were performed and animal-based indicators (e.g., BCS, cleanliness, overgrown claws) were collected. Group-level observations were made in 1 single pen containing only adult lactating goats, with all animals being evaluated. If more pens were present, the pen to be assessed was chosen as the pen considered at higher risk for animal welfare (e.g., with higher stocking density or lower feeding space; Table 1). Even though the number of animals per pen varied across farms, no statistical differences related to farm size were found $(P$ $>0.05$ ). For the individual assessment, several animals were caught following the sampling strategy developed by Welfare Quality for dairy cows (Welfare Quality, 2009). This strategy included the inspection of several animals proportional to the pen size, with percentages ranging from $100 \%$ of the subjects (in pens with fewer than 30 animals) to a minimum of $25 \%$ of the subjects (in the pens with more than 150 animals), assuming a $50 \%$ prevalence, and considering a $90 \%$ interval of confidence and an accuracy of $10 \%$. Six assessors were involved in the trial in both countries, all being submitted to equal training, consisting of classroom presentations and exercises and practical field assessments.

\section{Data Management and Statistical Analysis}

Data were entered, compiled and statistically analyzed using SPSS version 23 (IBM SPSS Statistics, Armonk, NY). To perform an overall analysis, the prevalence of each animal-based indicator under study was calculated at farm level. Prevalence was expressed as number of animals affected in the pen divided by the total number of animals observed in the pen. Queuing at feeding and drinking indicators were recorded as the proportion of animals queuing on the total of animals in the pen. Goats' response toward AD test was expressed as the proportion of goats accepting to enter in contact with the assessor (contact) and accepting to be gently stroked (acceptance; Mattiello et al., 2010). Latency to the first contact test was expressed in seconds.

Four statistical methods were used to evaluate COT of the animal-based indicators (Figure 1). First, a Wilcoxon signed rank test was carried out to test whether the results obtained during the 2 visits were significantly different at the 0.05 level. Afterward, the indicators presenting nonsignificant differences between visits were evaluated following a discriminative $\left(\mathrm{r}_{\mathrm{s}}, \mathrm{ICC}\right)$ and an evaluative approach (LoA). The reliability of indicators presenting a prevalence value lower than $0.1 \%$ at least in 1 assessment was assumed to be meaningless (Hoehler, 2000; Plesch et al., 2010), and hence not ad-

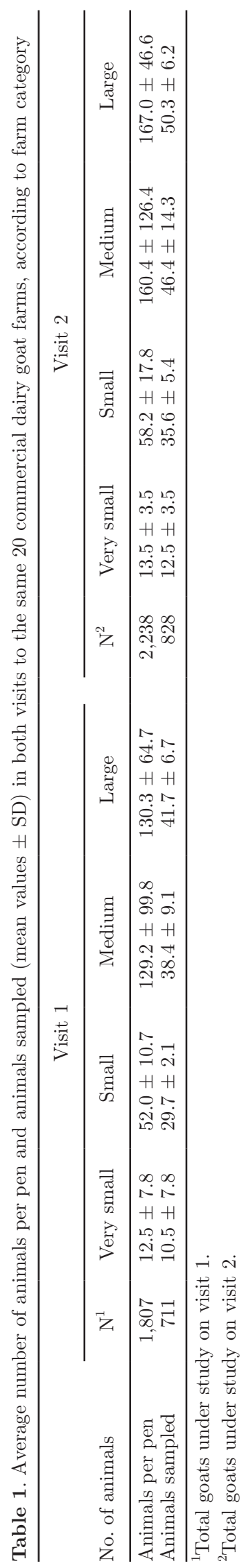




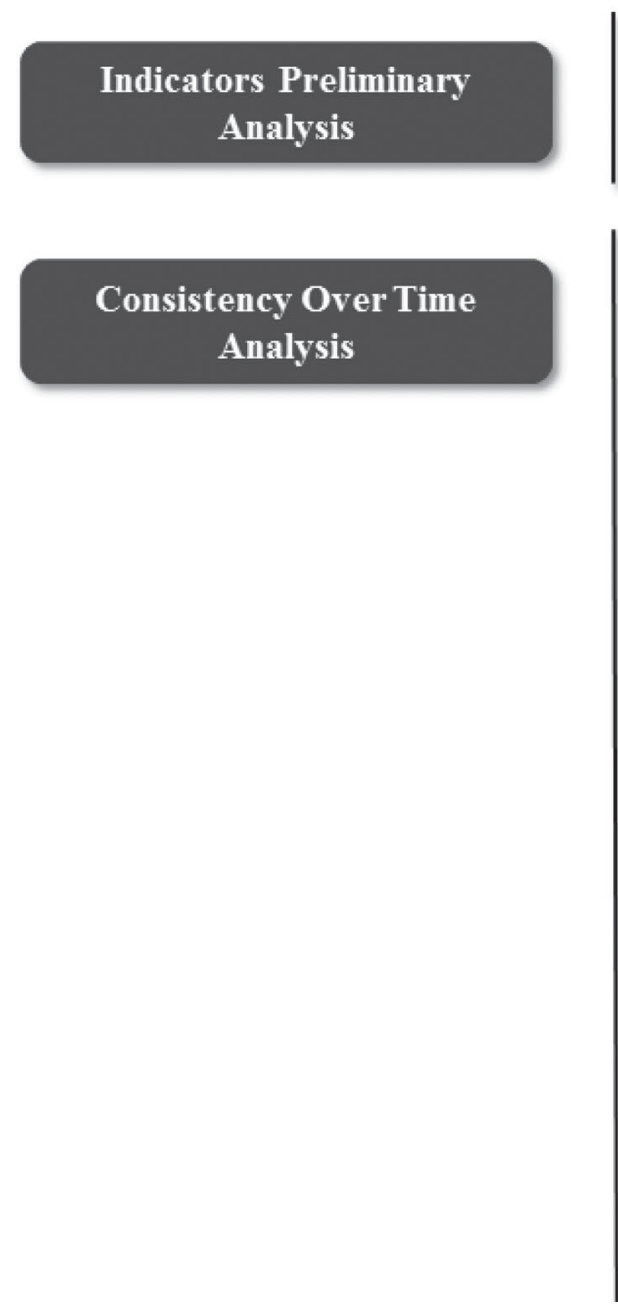

1

Prevalence/Proportion of each animal-based indicator per visit

2

Wilcoxon signed test between the results of the two assessments $(P<0.05)$

3

Discriminative approach

Evaluative approach

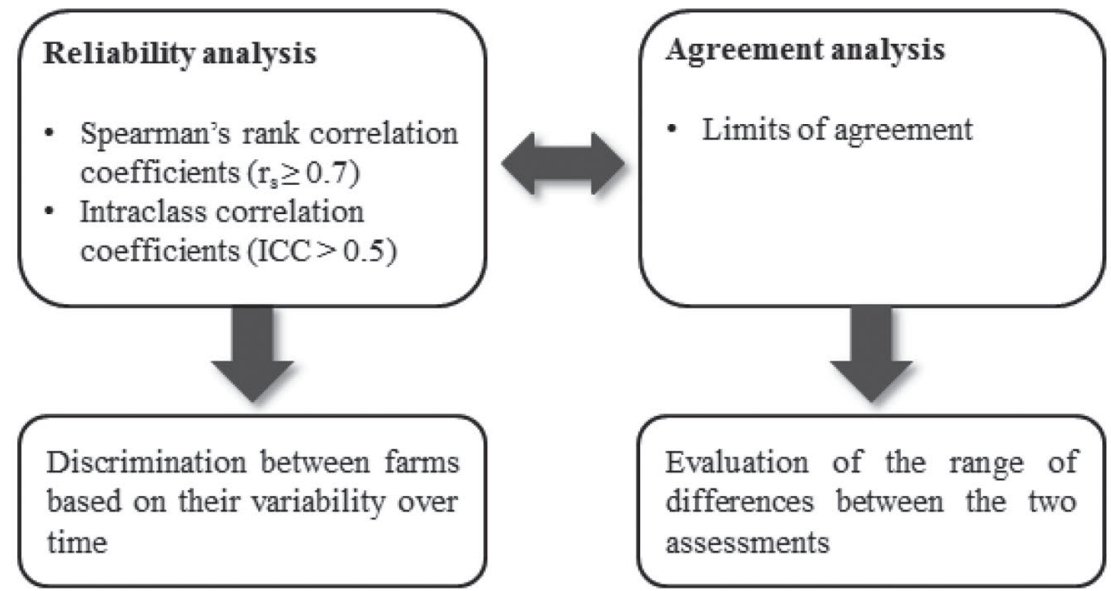

Figure 1. Statistical methods used to evaluate the consistency of indicators over time.

dressed. All the other indicators included in the AWIN prototype protocol for dairy goats, except for qualitative behavior assessment, were submitted to analysis.

Discriminative Approach. Spearman's rank correlation coefficients and ICC between the 2 visits were used to determine COT from a discriminative point of view, as they provide information on the ability of the results to distinguish between farms. Spearman's correlation coefficient evaluates the degree to which a set of measurements varies linearly with another set, but it does not directly compare the values obtained, as it ignores the scales of each set of measurements (Dohoo et al., 2009). Intraclass correlation coefficients, on the other hand, relate the measurement error to the variability between study objects and are considered the most appropriate and commonly used reliability measure for continuous variables (de Vet et al., 2006; Terwee et al., 2007). Intraclass correlation coefficients describe the variation between farms divided by the total variation (i.e., variation between farms plus the variation between visits to each farm; Terwee et al., 2007) and can be considered as 1 minus the proportion of variance that results from measurement error (Dohoo et al., 2009), which is related to the variability between farms. To reach a good reliability by following a discriminative approach, the measurement error of the welfare assessment system should be small when compared with the variation between farms (de Vet, 1998).

Intraclass correlation coefficients were calculated with a 2-way mixed effects model (Shrout and Fleiss, 1979); that is, the subjects in the study were considered to be random but the observers were not random effects, with absolute agreement and single-measures being estimated. Consistency over time of the animalbased indicators was considered acceptable if the Spear- 
man's correlation coefficients were equal or exceeded the threshold of 0.7 (Martin and Bateson, 2007) and if the variance explained by the random factor (variance between farms) was higher than the variance of the residuals describing the variance within farm (i.e., if the ICC were higher than 0.5; Dohoo et al., 2009).

Evaluative Approach. To determine COT from an evaluative perspective, the LoA between the 2 visits for each animal-based indicator were determined following the LoA method proposed by Bland and Altman (1986), which is not affected by the various sources of variability in the measurement setting. This method specifies the range of differences between 2 sets of measurements, being useful to determine if the level of disagreement between the 2 sets varies with the mean value of the variable being assessed (de Vet, 1998; Dohoo et al., 2009).

Limits of agreement were estimated on the mean difference of prevalences between the 2 visits to the same 20 dairy goat farms and the standard deviation of these differences, where approximately $95 \%$ of these differences are expected to lie between their lower and upper limits (mean differences $\pm 2 \mathrm{SD}$ of these differences). The smaller the range between these limits the better the agreement. The Bland and Altman (1986) plots of the differences between the pairs of prevalences against their mean help to determine the range of errors (de Vet et al., 2006), thus being useful to interpret the results.

\section{RESULTS}

\section{Preliminary Data Analysis and Wilcoxon Signed Rank Test}

The mean prevalences and proportions of the indicators assessed during the 2 visits are shown in Table 2. Most of the indicators presenting the highest (e.g., improper disbudding, overgrown claws) and the lowest prevalences (e.g., oblivion, abnormal lying) in the first set of visits also showed similar values in the second visit. Panting and shivering with a score of 2 were never recorded during both visits. Oblivion, abnormal lying, shivering with a score of 1 , vulvar discharge, and kneeling at the feeding rack were observed at prevalences of less than $0.1 \%$ in at least 1 visit, and so were not further considered for analysis.

According to the Wilcoxon signed rank test, grouplevel indicators such as hair coat condition, panting with a score of 1 , shivering with a score of 1 , and contact in $\mathrm{AD}$ presented prevalence results significantly different between visits at the 0.05 level. Regarding individual assessment, only very fat BCS was significantly different between visits (Table 2). The following indicators did not differ significantly between the 2 visits: improper disbudding, Queuing, acceptance in AD, severe lameness, and kneeling in the pen at group level; and very thin BCS, fecal soiling, udder asymmetry, cleanliness, abscesses, lesions, knee calluses, ocular discharge, nasal discharge, and overgrown claws at individual level.

\section{Discriminative Approach}

Body abscesses $\left(\mathrm{r}_{\mathrm{s}}=0.7\right.$; ICC $\left.=0.7\right)$ and cleanliness of the lower legs $\left(r_{\mathrm{s}}=0.7\right.$; ICC $\left.=0.7\right)$ presented Spearman's correlation coefficients equal or higher than the threshold of 0.7 and ICC higher than 0.5 at the 0.05 level, thus showing an acceptable reliability (Table 3 ). According to ICC, head lesions ( $\mathrm{ICC}=0.5 ; P=$ $0.029)$ and acceptance of $\mathrm{AD}(\mathrm{ICC}=0.8 ; P<0.001)$ showed good reliability between visits; however, their Spearman's correlations showed poor reliability $\left(\mathrm{r}_{\mathrm{s}}<\right.$ 0.7). Based on Spearman's correlations, the remaining indicators were not reliable between visits from a discriminative perspective (Table 3 ).

\section{Evaluative Approach}

The LoA for the mean differences between visits for the aforementioned indicators are presented in Table 3. Limits of agreement were plotted for body abscesses, cleanliness of the lower legs, head lesions, and acceptance in $\mathrm{AD}$ (Figures 2-5) as a complementary approach to guide the interpretation of the results obtained from Spearman's correlation coefficients and ICC.

The range of prevalence difference of body abscesses between visits oscillated from -12.4 to $15 \%$ for 19 of the 20 farms (Figure 2). Three farms presented almost similar results in both visits and the other 6 farms presented a 0 prevalence of body abscesses in both visits. Figure 3 shows the prevalence difference plot of cleanliness of the lower legs, which varied from -5 to $15 \%$ between the 2 visits for 15 of the 20 farms. One farm presented equal prevalence values in both visits $(\approx 6.3 \%)$, representing a complete agreement. Four farms presented almost similar results in both visits and 4 other farms presented a 0 prevalence of lower legs dirtiness in both visits. The prevalence difference of head lesions varied from -83.6 to $18.2 \%$ (Figure 4 ). Head lesions did not present any agreement, except for 2 farms that showed almost similar prevalences in both visits. Finally, the plot of the differences between the pairs of prevalences for acceptance in $\mathrm{AD}$ against the average prevalence of the 2 visits is illustrated in Figure 5 , showing that the scatter of the difference increases with the combined mean. Also, the range of prevalence difference between the 2 visits varied from -5 to $5 \%$ 


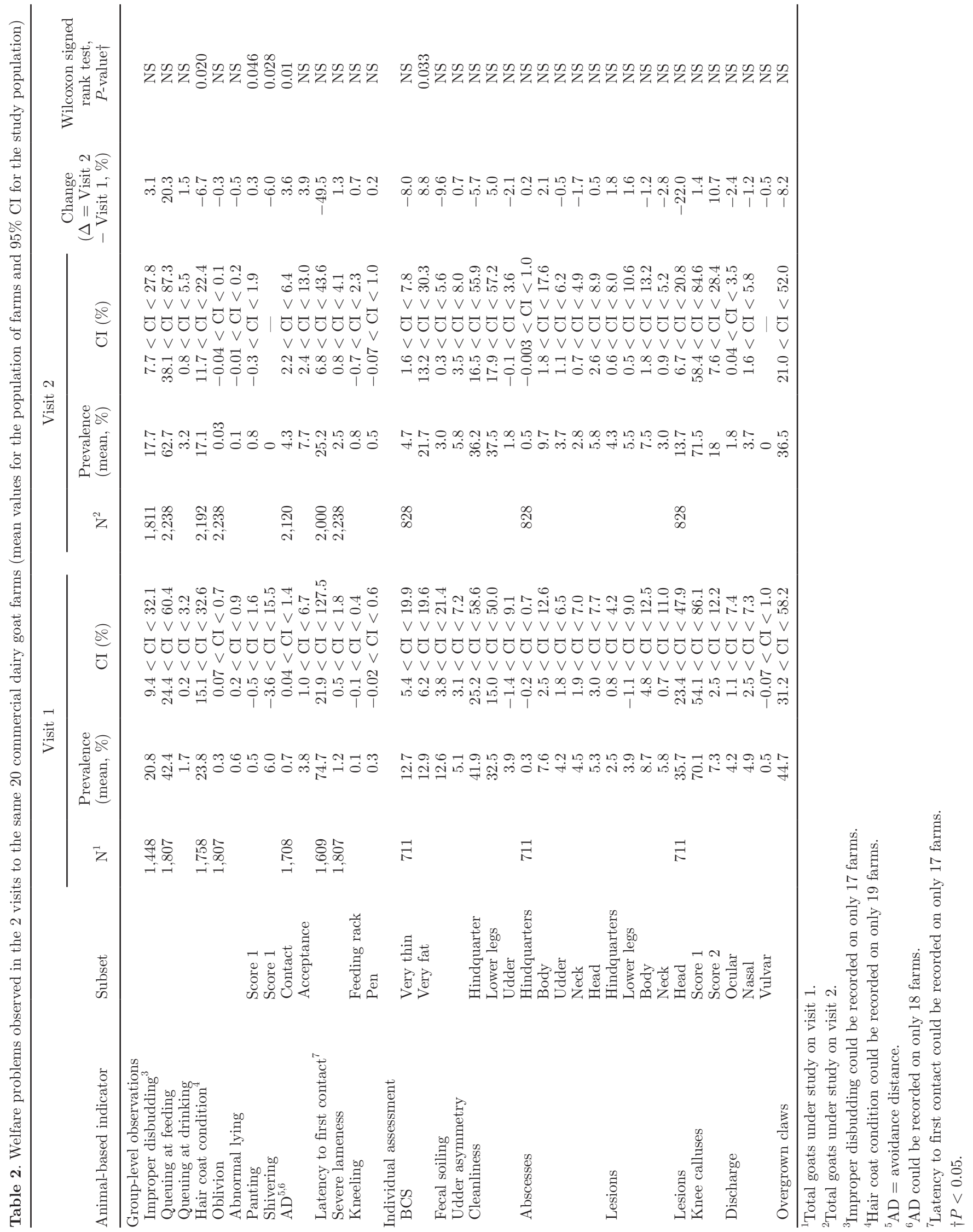




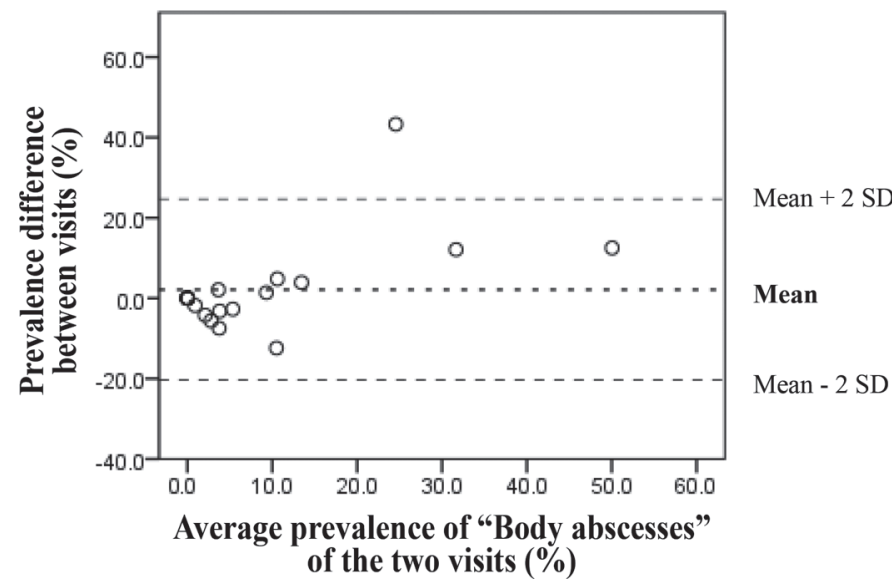

Figure 2. Plot of prevalence difference (second minus first visit) against the mean prevalence of body abscesses of the 2 visits at the same 20 farms $(\bigcirc$; mean differences between visits $\pm 2 \mathrm{SD})$. Six farms presented $0 \%$ prevalence of body abscesses in both visits.

for 15 of the 20 farms assessed, with 3 farms presenting a 0 prevalence.

\section{DISCUSSION}

To investigate COT of animal-based indicators for dairy goats we followed Temple et al. (2013) study that provides both a discriminative and an evaluative approach to the analysis of data.

\section{Discriminative Approach}

Spearman's rank correlation coefficients and ICC between the 2 visits were used to determine COT from

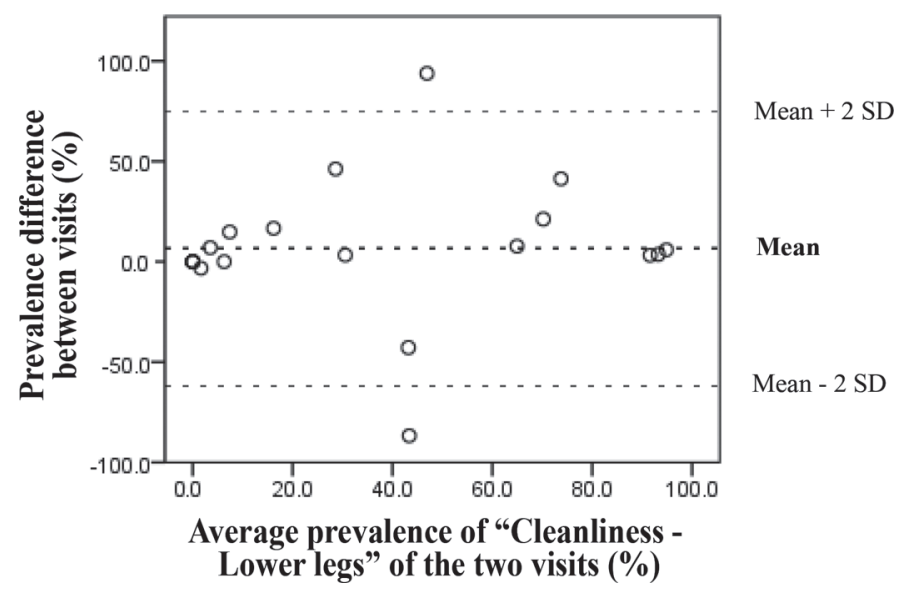

Figure 3. Plot of prevalence difference (second minus first visit) against the mean prevalence of cleanliness of the lower legs of the 2 visits at the same 20 farms $(O$; mean differences between visits \pm 2 $\mathrm{SD})$. Four farms presented $0 \%$ prevalence of lower legs dirtiness in both visits.

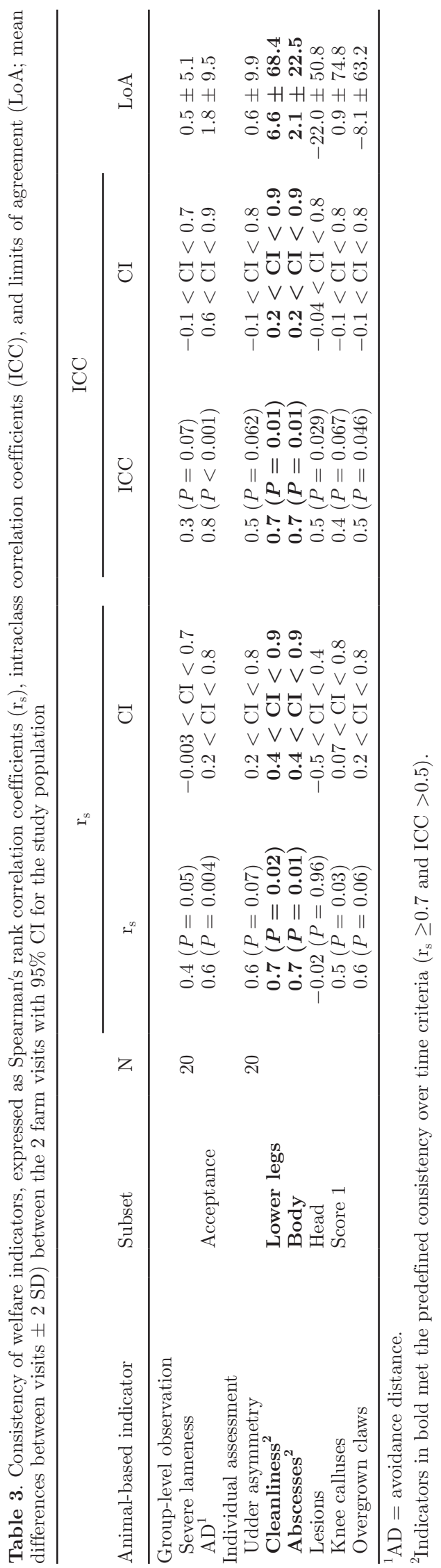




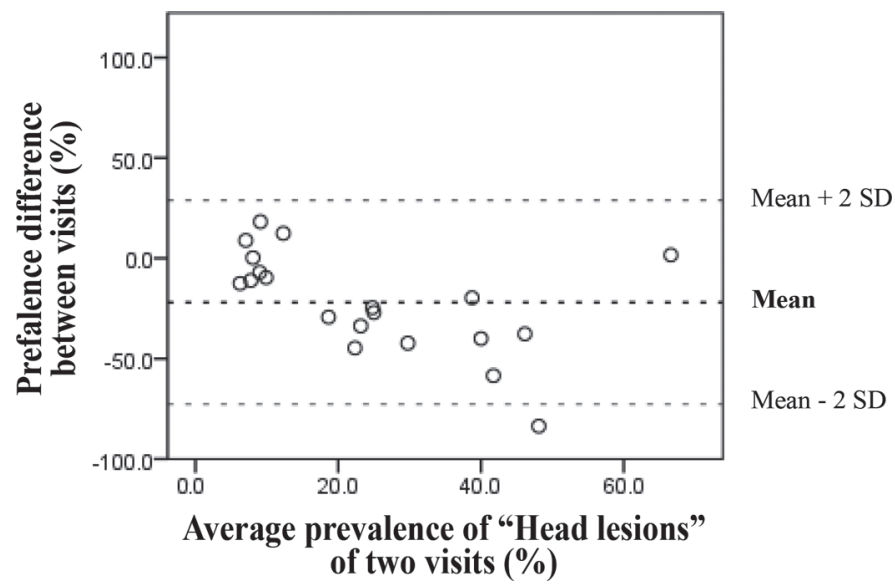

Figure 4. Plot of prevalence difference (second minus first visit) against the mean prevalence of head lesions of the 2 visits at the same 20 farms ( $\bigcirc$; mean differences between visits $\pm 2 \mathrm{SD})$.

a discriminative perspective. Only body abscesses and cleanliness of the lower legs were completely consistent between visits at the 0.05 level.

Body abscesses, mostly due to Caseous Lymphadenitis, are typically chronic conditions that persevere, supporting the consistency of this indicator over time. Caseous Lymphadenitis is recognized as an endemic disease in many countries (Williamson, 2001), and field studies in Portugal and Italy showed the presence of abscesses in $90 \%$ of the visited farms (Battini et al., 2016; Can et al., 2016). These findings support the inclusion of this indicator in a welfare assessment protocol, as it

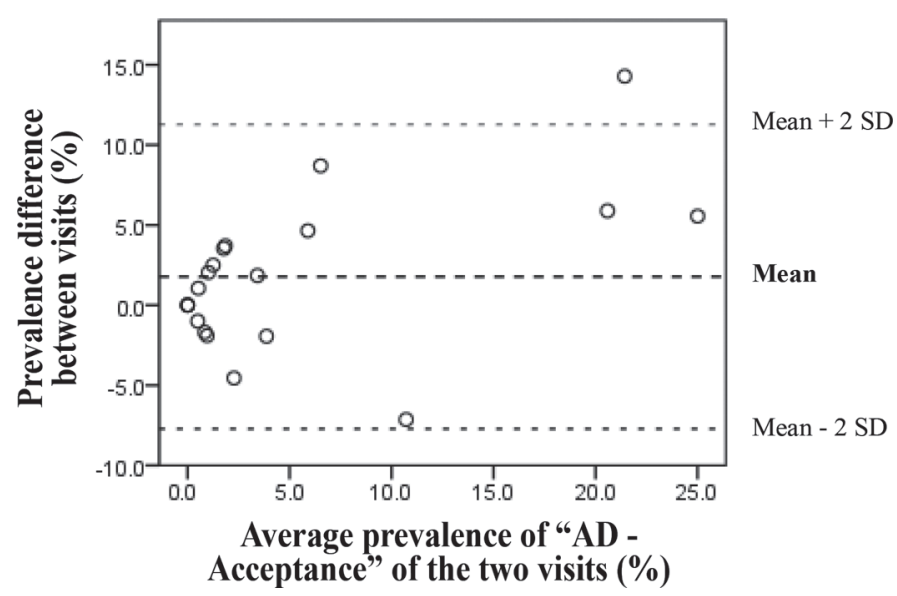

Figure 5. Plot of prevalence difference (second minus first visit) against the mean prevalence of avoidance distance (AD) acceptance of the 2 visits at the same 20 farms $(\bigcirc$; mean differences between visits $\pm 2 \mathrm{SD})$. Three farms presented $0 \%$ prevalence of acceptances in both visits. allows the identification of a widespread welfare problem (Ferrante et al., 2012).

Regarding cleanliness of the lower legs, goats' dirtiness is mostly influenced by cleaning routines. Lower legs dirtiness was also a highly prevalent welfare problem in both Portuguese and Italian farms (Battini et al., 2016; Can et al., 2016), and this might explain the high consistency of this indicator. However, during the on-farm testing of the prototype the visual assessment of cleanliness was greatly influenced by the animals' coat color, reflecting the limited validity and reliability of such indicator (Battini et al., 2015, 2016; Can et al., 2016); therefore, this measure replaced by a resourcebased indicator (bedding) in the final AWIN protocol for dairy goats (AWIN, 2015).

Head lesions showed a nonsignificant low Spearman's correlation coefficient between visits, but an acceptable ICC at the 0.05 level. The higher value of the ICC can be explained by the higher variability between farms in comparison to the variation between visits to each farm. Actually, the on-farm testing of head lesions resulted in some difficulties related to goats' breed and molting stage, which gave rise to low levels of reliability (Battini et al., 2015; A. Vieira, unpublished data), contributing to its elimination from the final protocol.

Similar conflicting results between Spearman's correlation coefficients and ICC were observed for acceptance of $\mathrm{AD}$ at the significance level of 0.05 . This consideration, together with the problems related to its possible variation with the breed in assessment and to its questionable feasibility, lead to the elimination of the $\mathrm{AD}$ test from the final protocol (Battini et al., 2015; Can et al., 2016).

Severe lameness, udder asymmetry, overgrown claws, and knee calluses with a score of 1 showed Spearman's correlation coefficients and ICC estimates near 0.7 and 0.5 , respectively, trending toward statistical significance $(P<0.05)$, which can indicate almost consistent results between visits. As a result, these indicators were all considered for inclusion in the AWIN welfare assessment protocol with slight adjustments (Battini et al., 2015, 2016; Can et al., 2016). This approach allowed us to discriminate between farms based on their variability over time; nevertheless, considering the observed dependence of Spearman's coefficients and ICC on the variance of the recorded population of farms, a complementary analysis is required.

\section{Evaluative Approach}

The LoA calculated in our study are correct in that they are expected to include $95 \%$ of differences; however, some particular conclusions can be drawn. For 
instance, the Bland-Altman plot shows that for body abscesses the LoA are too wide at the low prevalences end and too narrow for higher prevalences. For cleanliness of the lower legs, the LoA are too narrow for average prevalences and too wide for the rest of farms. Furthermore, the wider LoA of head lesions can be explained by the large values of the prevalence difference between visits for high average prevalences, showing that this indicator is not consistent over time for high prevalences. For acceptance of $\mathrm{AD}$, the scatter of the prevalence differences increases as the average prevalence increases, meaning that this indicator is also not consistent over time for high prevalences.

For many indicators with high prevalence, the mean difference between the pairs of prevalences was near 0 , meaning that the farms present these welfare issues persistently, thus requiring further attention. For instance, chronic conditions, such as asymmetric udders, overgrown claws, severe lameness, and knee calluses, were not expected to appear as outbreaks; thus, following this evaluative approach can be a practical way of identifying farms that show persistent welfare issues, being at a higher risk of poor welfare, which might also be useful for improvement purposes.

\section{Overall Analysis and Considerations}

Several reasons may justify a low level of consistency. Blokhuis et al. (2013) suggested that the variability of an indicator across assessments may be caused by variation due to real differences of its prevalence, because of changes in management, feeding, or lactation stages (as for instance in the case of very fat BCS) or due to an outbreak of a specific disease (as for instance in the case of fecal soiling). Similarly, Temple et al. (2013) pointed out that the variability of an indicator can be explained by seasonal effects, which can justify the significantly different results of a score of 1 for panting and shivering between visits. Such indicators are expected to show a low reliability over time, as their prevalence might vary in specific months or years.

Even though no major changes in management or housing occurred during the period between the 2 visits, we cannot overrule that management routines might have slightly changed in some farms; for instance, the lack of consistency of indicators related to the criteria of good health (e.g., nasal discharge, hair coat condition) may be explained by a proper management of the sick animals and the adoption of management or disease control measures by the farmer. These findings show that some indicators might be too sensitive and their variation might be related to slight alterations in the farm environment or in the animals themselves.
Methodological constraints, such as intraobserver effect, sample strategy, and sample size, might also be responsible for a low COT. Similarly, indicators with low prevalences (e.g., oblivion, kneeling at the feeding rack) often show low COT, as a population with a small proportion of affected animals will deliver artificially low reliability values (Hoehler, 2000; Plesch et al., 2010).

Even though this analysis of data from only 2 visits is unlikely to represent accurately the real variability over a long period of time, it can still provide some guidance on the variability of animal-based indicators.

Capdeville and Veissier (2001) and Winckler et al. (2007) reported that high levels of consistency are essential for welfare assessment systems at farm level, guaranteeing objectivity for the farmer and system's credibility. In this sense, COT is particularly relevant when welfare assessment is to be used for certification purposes in terms of welfare labeling (Blokhuis et al., 2013), allowing farmers to keep track of change over time and discriminating between farms. However, welfare assessment schemes may have several other objectives rather than to discriminate between farms, such as advising farmers on animal health and welfare improvement, checking compliance with legislative requirements, or comparing systems to improve legislation (Botreau et al., 2007b).

In addition, indicator COT also contributes to the reduction of recording costs (Sørensen et al., 2007; Knierim and Winckler, 2009), as indicators that do not change significantly over a long period of time will not require regularly repeated visits to obtain reliable estimates. This is in line with the AWIN stepwise approach (AWIN, 2015); that is, in a first-level welfare assessment a quick screening of the farm is performed by recording highly consistent and feasible indicators (e.g., abscesses). This first level allows the discrimination between farms based on the identification of persistent welfare problems; those farms not reaching an acceptable outcome (e.g., in the worst $5 \%$ of the reference population for 1 indicator) or not complying with the current legislation are then targeted for a second-level assessment. This second level entails a more comprehensive protocol involving the recording of indicators more likely to be subject to variations along time, such as fecal soiling, BCS, and nasal discharge.

Due to the fact that the AWIN welfare assessment protocol for dairy goats is fairly new and under continuous improvement and revision, repeated assessments and long-term studies on the effects of different sample sizes and appropriate number, interval, and schedule of assessments appear to be necessary to provide further information on which indicators offer reliable results with long-term collection and which are most likely to 
drive change. This will help to determine the frequency of visits necessary to obtain a consistent and feasible welfare assessment scheme.

\section{CONCLUSIONS}

From a discriminative perspective, only body abscesses and cleanliness of the lower legs were consistent over the 3 -mo period. The remaining indicators failed to fulfil at least 1 of the 2 predefined consistency criteria even though some indicators presented close results. The LoA method was a useful complementary approach to guide the interpretation of indicator COT, helping to identify farms with persistent welfare issues, being practical for improvement purposes. The results obtained by the combined use of these statistical methods allowed us to reach conclusions that contributed to the outlining of the AWIN welfare assessment protocol for dairy goats, proving to be a sound approach to overcome the statistical inconsistencies reported in the literature.

\section{ACKNOWLEDGMENTS}

The Animal Welfare Indicators (AWIN) project (FP7-KBBE-2010- 4) has received funding from the European Union 7th Framework Programme for research, technological development, and demonstration, and CIISA Project UID/CVT/00276/2013 was supported by CIISA-FMV-Universidade de Lisboa (Lisbon, Portugal). We also acknowledge the support of the Division of Agricultural and Environmental Sciences, University of Nottingham (Loughborough, UK).

\section{REFERENCES}

AWIN. 2015. AWIN welfare assessment protocol for goats. Accessed Jan. 23, 2017. http://dx.doi.org/10.13130/AWIN_goats_2015.

Battini, M., S. Barbieri, A. Vieira, G. Stilwell, and S. Mattiello. 2016. Results of testing the prototype of the AWIN welfare assessment protocol for dairy goats in 30 intensive farms in Northern Italy. Ital. J. Anim. Sci. 15:283-293. https://doi.org/10.1080/1828051X .2016.1150795.

Battini, M., G. Stilwell, A. Vieira, S. Barbieri, E. Canali, and S. Mattiello. 2015. On-farm welfare assessment protocol for adult dairy goats in intensive production systems. Animals (Basel) 5:934-950. https://doi.org/10.3390/ani5040393.

Bland, J. M., and D. G. Altman. 1986. Statistical methods for assessing two methods of clinical measurement. Lancet 327:307-310. https://doi.org/10.1016/S0140-6736(86)90837-8.

Blokhuis, H., M. Miele, I. Veissier, and B. Jones, ed. 2013. Improving farm animal welfare. Science and society working together: The Welfare Quality approach. Wageningen Academic Publishers, Wageningen, the Netherlands.

Bokkers, E. A. M., H. Leruste, L. F. M. Heutinck, M. Wolthuis-Fillerup, J. T. N. van der Werf, B. J. Lensink, and C. G. Van Reenen. 2009. Inter-observer and test-retest reliability of on-farm behavioural observations in veal calves. Anim. Welf. 18:381-390.
Botreau, R., M. Bonde, A. Butterworth, P. Perny, M. Bracke, J. Capdeville, and I. Veissier. 2007b. Aggregation of measures to produce an overall assessment of animal welfare. Part 1: A review of existing methods. Animal 1:1179-1187. https://doi.org/10.1017/ S1751731107000535.

Botreau, R., I. Veissier, A. Butterworth, M. Bracke, and L. Keeling. 2007a. Definition of criteria for overall assessment of animal welfare. Anim. Welf. 16:225-228.

Can, E., A. Vieira, M. Battini, S. Mattiello, and G. Stilwell. 2016. Onfarm welfare assessment of dairy goat farms using animal-based indicators: The example of 30 commercial farms in Portugal. Acta Agric. Scand. A Anim. Sci. 66:43-55. https://doi.org/10.1080/ 09064702.2016.1208267.

Capdeville, J., and I. Veissier. 2001. A method of assessing welfare in loose housed dairy cows at farm level, focusing on animal observations. Acta Agric. Scand. A Anim. Sci. 51(Suppl. 30):62-68. https://doi.org/10.1080/090647001316923081.

Costa-Santos, C., J. Bernardes, D. Ayres-de-Campos, A. Costa, and C. Costa. 2011. The limits of agreement and the intraclass correlation coefficient may be inconsistent in the interpretation of agreement. J. Clin. Epidemiol. 64:264-269. https://doi.org/10.1016/j.jclinepi 2009.11.010.

Czycholl, I., C. Kniese, K. Büttner, E. Grosse Beilage, L. Schrader, and J. Krieter. 2016. Test-retest reliability of the Welfare Quality animal welfare assessment protocol for growing pigs. Anim. Welf. 25:447-459. https://doi.org/10.7120/09627286.25.4.447.

de Vet, H. C. W. 1998. Observer reliability and agreement. Pages 3123-3128 in Encyclopedia of Biostatistics P. Armitage and T. Colton, ed. John Wiley and Sons, New York, NY.

de Vet, H. C. W., C. B. Terwee, D. L. Knol, and L. M. Bouter. 2006. When to use agreement versus reliability measures. J. Clin. Epidemiol. 59:1033-1039. https://doi.org/10.1016/j.jclinepi.2005.10 .015 .

Dohoo, I., W. Martin, and H. Stryhn. 2009. Screening and diagnostic tests. Pages 91-134 in Veterinary Epidemiological Research I. Dohoo, W. Martin, and H. Stryhn, ed. VER Inc, Charlottetown, Prince Edward Island, Canada.

Ferrante, V., M. Battini, C. Caslini, L. Grosso, E. Mantova, L. Noé, S. Barbieri, and S. Mattiello. 2012. Presence of abscesses as a welfare indicator in dairy goats: A preliminary study. Page 224 in Proc. of XLVI Congress of the International Society for Applied Ethology. Wien, Austria. Wageningen Academic Publishers, Wageningen, the Netherlands.

Guyatt, G., S. Walter, and G. Norman. 1987. Measuring change over time: Assessing the usefulness of evaluative instruments. J. Chronic Dis. 40:171-178. https://doi.org/10.1016/0021-9681(87)90069-5.

Hoehler, F. K. 2000. Bias and prevalence effects on kappa viewed in terms of sensitivity and specificity. J. Clin. Epidemiol. 53:499-503. https://doi.org/10.1016/S0895-4356(99)00174-2.

Kirchner, M. K., H. Schulze Westerath, U. Knierim, E. Tessitore, G. Cozzi, and C. Winckler. 2014. On-farm animal welfare assessment in beef bulls: Consistency over time of single measures and aggregated Welfare Quality scores. Animal 8:461-469. https://doi.org/ $10.1017 /$ S1751731113002267.

Knierim, U., and C. Winckler. 2009. On-farm welfare assessment in cattle: Validity, reliability and feasibility issues and future perspectives with special regard to the Welfare Quality approach. Anim. Welf. 18:451-458.

Martin, P., and P. Bateson. 2007. Measuring Behaviour: An Introductory Guide. Cambridge University Press, Cambridge, UK.

Mattiello, S., M. Battini, E. Andreoli, M. Minero, S. Barbieri, and E. Canali. 2010. Avoidance distance test in goats: A comparison with its application in cows. Small Rumin. Res. 91:215-218. https://doi .org/10.3390/ani5040393.

O'Callaghan, K. A., P. J. Cripps, D. Y. Downham, and R. D. Murray. 2003. Subjective and objective assessment of pain and discomfort due to lameness in dairy cattle. Anim. Welf. 12:605-610.

Plesch, G., N. Broerkens, S. Laister, C. Winckler, and U. Knierim. 2010. Reliability and feasibility of selected measures concerning resting behaviour for the on-farm welfare assessment in dairy cows. 
Appl. Anim. Behav. Sci. 126:19-26. https://doi.org/10.1016/j .applanim.2010.05.003.

Scott, E. M., A. M. Nolan, and J. L. Fitzpatrick. 2001. Conceptual and methodological issues related to welfare assessment: A framework for measurement. Acta Agric. Scand. A Anim. Sci. 51(Suppl. 30):5-10. https://doi.org/10.1080/090647001316922983.

Shrout, P. E., and J. L. Fleiss. 1979. Intraclass correlations: Uses in assessing rater reliability. Psychol. Bull. 86:420-428.

Sørensen, J. T., T. Rousing, S. H. Møller, M. Bonde, and L. Hegelund 2007. On-farm welfare assessment systems: What are the recording costs? Anim. Welf. 16:237-239.

Streiner, D. L., and G. R. Norman. 2003. Health Measurement Scales: A Practical Guide to Their Development and Use. Oxford University Press, New York, NY.

Temple, D., X. Manteca, A. Dalmau, and A. Velarde. 2013. Assessment of test-retest reliability of animal-based measures on growing pig farms. Livest. Sci. 151:35-45. https://doi.org/10.1016/j.livsci .2012 .10 .012 .
Terwee, C. B., S. D. M. Bot, M. R. de Boer, D. A. W. M. van der Windt, D. L. Knol, J. Dekker, L. M. Bouter, and H. C. W. de Vet. 2007. Quality criteria were proposed for measurement properties of health status questionnaires. J. Clin. Epidemiol. 60:34-42. https:// doi.org/10.1016/j.jclinepi.2006.03.012.

Waiblinger, S., U. Knierim, and C. Winckler. 2001. The development of an epidemiologically based on-farm welfare assessment system for use with dairy cows. Acta Agric. Scand A. Anim. Sci. 51(Suppl. 30):73-77. https://doi.org/10.1080/090647001316923108.

Welfare Quality. 2009. Welfare Quality Assessment Protocol for Cattle. Welfare Quality Consortium, Lelystad, the Netherlands.

Williamson, L. H. 2001. Caseous lymphadenitis in small ruminants. Vet. Clin. North Am. Food Anim. Pract. 17:359-371. https://doi .org/10.1016/S0749-0720(15)30033-5.

Winckler, C., J. Brinkmann, and J. Glatz. 2007. Long-term consistency of selected animal-related welfare parameters in dairy farms. Anim. Welf. 16:197-199. 